\title{
Persistence of Antipsychotic Treatment in Elderly Dementia Patients: A Retrospective, Population-Based Cohort Study
}

\author{
Gavin Mast ${ }^{1} \cdot$ Kimberly Fernandes $^{2} \cdot$ Mina Tadrous $^{1,2,3} \cdot$ Diana Martins $^{2}$ • \\ Nathan Herrmann ${ }^{4,5}$ - Tara Gomes ${ }^{1,2,3,6}$
}

Published online: 13 May 2016

(c) The Author(s) 2016. This article is published with open access at Springerlink.com

\begin{abstract}
Background Antipsychotics are commonly used to manage behavioral and psychological symptoms of dementia. Concerns over their safety and efficacy in this role have resulted in antipsychotics typically being recommended for short-term usage only when used among dementia patients. However, there is little work examining the duration of antipsychotic treatment in the elderly dementia patient population.

Objective To determine the persistence of use of antipsychotics in elderly dementia patients and the role of dose on therapy duration.

Methods A retrospective, population-based cohort study using administrative data, including dispensing records from a provincial public drug program, from Ontario,
\end{abstract}

Tara Gomes

GomesT@smh.ca

1 St. Michael's Hospital, 30 Bond Street, Toronto, ON M5B 1W8, Canada

2 Institute for Clinical Evaluative Sciences, 2075 Bayview Avenue, Toronto, ON, Canada

3 Leslie Dan Faculty of Pharmacy, University of Toronto, 144 College Street, Toronto, ON, Canada

4 Hurvitz Brain Sciences Program, Sunnybrook Health Sciences Centre, 2075 Bayview Avenue, Toronto, ON, Canada

5 Faculty of Medicine, University of Toronto, 1 King's College Circle, Toronto, ON, Canada

6 Institute for Health Policy, Management and Evaluation, University of Toronto, 155 College Street, Suite 425, Toronto, ON, Canada
Canada between 2009 and 2012. Elderly dementia patients newly initiated onto antipsychotics were followed until drug discontinuation, death, 2-year follow-up, or end of study. Competing risk analysis was performed to determine time to discontinuation, stratified by categories of initial dose.

Results After 2 years $49.1 \%$ of the cohort $(N=22,927$ of 46,695) had discontinued treatment. When stratified by dose, the high-dose group (51.1\% discontinued) discontinued more frequently than the medium- $(48.7 \%$ discontinued) and low- (47.5\% discontinued) dose groups $(p<0.0001)$.

Conclusion Approximately half of elderly dementia patients treated with antipsychotics discontinue within 2 years, with those on higher doses more likely to discontinue. However, the number of patients remaining on therapy represents a serious public health concern.

\section{Key Points}

Antipsychotics are often used to manage the behavioral and psychological symptoms of dementia, although safety and efficacy concerns generally warrant only short-term usage of these medications.

Just over half of elderly dementia patients treated with antipsychotics in Ontario, Canada, were persistent to drug treatment during the 2-year study period.

Patients initiated on a higher initial dose of antipsychotic appeared to discontinue more frequently than those treated with lower doses. 


\section{Introduction}

Antipsychotics represent a drug class that has garnered a large amount of both clinical and public interest in its role in treating a number of mental health conditions, particularly with regard to the elderly. These drugs have been used for many years to treat psychosis-associated conditions such as schizophrenia and bipolar disorder; however, they are also used extensively to treat behavioral and psychological symptoms of dementia (BPSD) [1, 2]. As antipsychotics have been increasingly used to treat BPSD, their safety and efficacy has come under question. It is now well documented that the use of antipsychotics among the elderly, particularly those with dementia, results in increased morbidity and mortality [2-6]. Specifically, observational studies using administrative databases have demonstrated increased risk of cardiovascular death, heart failure, and sudden death, as well as a heightened risk for falls and fractures amongst elderly patients with dementia taking both typical and atypical antipsychotics $[1,4,5,7]$. This body of work has prompted governmental agencies, including the US Food and Drug Administration (FDA) and Health Canada, to advise against the use of antipsychotics in those with dementia, except for treating only the most severe behavioral and psychological symptoms of dementia $[8,9]$.

With the widespread use of antipsychotics and the various different drugs and formulations available, a deeper understanding of dose prescribed and duration of therapy among the elderly is important to explore. Among patients with schizophrenia and bipolar disorder, where antipsychotic use is indicated, studies have shown low rates of adherence to both typical and atypical antipsychotics [1014]. While the nature of these conditions may contribute to discontinuation, adverse effects of the drugs also appear to play a vital role in discontinuation of treatment [10-13]. Furthermore, many clinical practice guidelines recommend short-duration treatment with antipsychotics among dementia patients, recommending 3-6 months before discontinuing, with frequent monitoring to assess safety and efficacy [15].

Persistence to antipsychotic therapy has been explored in increasing detail among schizophrenia and other psychosis-associated patients, though there is little information regarding persistence rates in elderly patients with dementia, who differ from the other patients as a short duration of antipsychotic use is recommended. We examined elderly patients with dementia to identify trends in their persistence to antipsychotic therapy, and how duration of therapy is influenced by medication dose.

\section{Methods}

\subsection{Study Design}

We conducted a population-based, retrospective cohort study among patients with dementia aged 66 years or older residing in Ontario, Canada, and who were initiated on treatment with an antipsychotic between 1 January 2009 and 31 December 2012. This study was approved by the Research Ethics Board of Sunnybrook Health Sciences Centre, Toronto, ON, Canada.

\subsection{Data Sources}

We used linked administrative healthcare databases for cohort identification, co-morbidity assessment, and outcome ascertainment. We used the Ontario Drug Benefit (ODB) database, a computerized pharmacy records system that records prescription drugs dispensed to all Ontario residents over 65 years of age to obtain prescription data. The Canadian Institute for Health Information Discharge Abstract Database (CIHI-DAD) was used to obtain records of inpatient hospitalizations, including diagnostic and procedural information, while the CIHI National Ambulatory Care Reporting System (CIHI-NACRS) was used to obtain details of emergency department visits. Furthermore, the CIHI Ontario Mental Health Reporting System (CIHI-OMHRS) was used to obtain information about mental health diagnoses. Physician billing information and demographic information were obtained from the Ontario Health Insurance Plan (OHIP) database and the Ontario Registered Persons Database (RPDB), respectively. Data from the OHIP database was coupled with the ICES Physician Database (IPDB) to identify the specialty of antipsychotic prescribers. These datasets were linked using unique, encoded identifiers and analyzed at the Institute for Clinical Evaluative Sciences (ICES).

\subsection{Cohort Definition}

We identified users of antipsychotics based upon prescription records for any atypical or typical antipsychotic within the accrual period (January 2009 to December 2012). We defined the date of each patient's first prescription as their cohort entry date and restricted the cohort to patients with dementia by means of a validated dementia definition, based on a hospitalization or physician visit with a dementia diagnosis in the previous 5 years (using OHIP or CIHI-DAD diagnostic codes) or a cognitive enhancer prescription dispensed in the year prior [16]. We excluded patients less than 66 years old at cohort entry and those 
who were prescribed any antipsychotic in the year prior to the cohort entry date in order to restrict the cohort to elderly individuals newly initiated on antipsychotic treatment. Finally, individuals were excluded if they were only dispensed one prescription, as it is highly likely that these individuals never initiated therapy. This is an approach used regularly in research leveraging administrative claims databases investigating medication persistence $[17,18]$.

\subsection{Duration of Exposure}

Patients were followed forward from the date of their first prescription until they discontinued therapy, died, had 2 years of follow-up, or reached the end of the study period (31 December 2013). For each patient, a period of continuous drug use was defined as receipt of a subsequent prescription refill within 180 days of the previous prescription in order to account for varied prescription regimes and minor lapses in treatment. Patients who switched between different antipsychotics were still considered to be persistent. In a sensitivity analyses, we used an alternate, stricter definition for continuous use, whereby an individual was persistent if a prescription refill was identified within 1.5 times the days supplied of the previous prescription.

\subsection{Statistical Analyses}

We summarized the baseline characteristics of all individuals included in the study, including demographic information and a variety of relevant clinical characteristics. We also examined the prevalence of schizophrenia and bipolar co-diagnoses among patients in our cohort by looking for relevant diagnostic codes from CIHI-OMHRS and OHIP databases [19]. We conducted a competing risk analysis to determine time to discontinuation of any antipsychotic, accounting for death as a competing risk as a potentially high mortality rate was hypothesized for our cohort. We reported all analyses overall and stratified by the antipsychotic dose initiated to observe the effect of dosage on persistence. Doses were divided into three categories defined using tertiles of the dose initiated-low dose ( $<26 \mathrm{mg}$ /day), medium dose (26-74 mg/day), and high dose ( $>74 \mathrm{mg} /$ day -normalized into milligrams of chlorpromazine equivalent dose [20]. Standardized differences were calculated to compare the characteristics of the low- and medium-dose groups against the high dose group, with significance achieved if the standardized difference was greater than 0.1 [21]. Gray's test was used to check for the significance of any differences in the time to discontinuation between dose groups in the competing risk analysis [22]. All analyses were conducted using SAS Enterprise Guide (version 6.1, Cary, NC, USA).

\section{Results}

\subsection{Patient Characteristics}

Over the study period, we identified 60,594 elderly individuals with dementia who were newly initiated onto antipsychotic treatment, among whom 46,695 individuals were dispensed more than one antipsychotic prescription and so were eligible for the study. Of those eligible, 16,563 (35.5\%) initiated therapy on low-dose, 14,266 (30.6\%) initiated therapy on medium-dose, and 15,866 (34.0\%) initiated therapy on high-dose antipsychotic therapy. The median age for the new patients beginning treatment was 84 years (interquartile range 78-88), and 29,064 (62.2\%) of the patients were women. We found that overall only $1.6 \%$ of individuals had a co-diagnosis of schizophrenia, and $0.8 \%$ had a co-diagnosis of bipolar disorder. The patient characteristics are shown in Table 1, while the number of patients initiated on specific antipsychotics is shown in Table 2.

\subsection{Primary Analyses}

Of the 46,695 elderly patients with dementia who newly initiated antipsychotic therapy, $24.4 \%$ discontinued therapy after 6 months and $35.1 \%$ discontinued therapy after 1 year. By the end of the 2-year study period, $49.1 \%$ of patients in the cohort had discontinued antipsychotic therapy. Furthermore, we observed that $18.4 \%$ of patients had died by the end of the study. The results from the competing risk analysis are shown in Fig. 1.

When we stratified by the initial dose, we found that after a 1-year follow-up, $32.3 \%$ of low-dose patients, $34.1 \%$ of medium-dose patients, and $39.0 \%$ of highdose patients had discontinued antipsychotic therapy. Similarly, by the end of the study period, $47.5 \%$ of lowdose patients, $48.7 \%$ of medium-dose patients, and $51.1 \%$ of high-dose patients had discontinued antipsychotic therapy, while $17.0 \%$ of low-dose patients, $17.6 \%$ of medium-dose patients, and $20.7 \%$ of highdose patients had died (Fig. 2). The difference in time to discontinuation across the dose levels was found to be significant in our primary analysis $(p<0.0001)$. In our sensitivity analysis using the stricter definition of continuous use, the results were consistent $(p<0.0001)$. The mean duration of follow-up for all individuals in the cohort was 393 days [standard deviation (SD) 273 days] .The mean duration of antipsychotic use among those starting on a low dose was 258 days (SD 203 days), compared with 246 days (SD 200 days) and 213 days (SD 193 days) among those initiating medium- and highdose therapy, respectively. 
Table 1 Baseline characteristics of elderly dementia patients newly initiated on antipsychotic treatments

\begin{tabular}{|c|c|c|c|c|}
\hline & \multirow{2}{*}{$\begin{array}{l}\text { Overall } \\
N=46,695\end{array}$} & \multicolumn{3}{|c|}{ Index equivalent daily dose } \\
\hline & & $\begin{array}{l}\text { Low }(\leq 25 \mathrm{mg}) \\
N=16,563\end{array}$ & $\begin{array}{l}\text { Medium }(>25-74 \mathrm{mg}) \\
N=14,266\end{array}$ & $\begin{array}{l}\text { High }(>74 \mathrm{mg}) \\
N=15,866\end{array}$ \\
\hline \multicolumn{5}{|l|}{ Age and sex } \\
\hline Median (IQR) & $84(78-88)$ & $84(79-89)^{*}$ & $84(78-88)^{*}$ & $83(77-88)$ \\
\hline Male $(\%)$ & $17,631(37.8 \%)$ & $5843(35.3 \%)^{*}$ & $5296(37.1 \%)$ & $6492(40.9 \%)$ \\
\hline \multicolumn{5}{|l|}{ Location of residence } \\
\hline Missing & $67(0.1 \%)$ & $16(0.1 \%)$ & $23(0.2 \%)$ & $28(0.2 \%)$ \\
\hline Urban & $40,295(86.3 \%)$ & $14,637(88.4 \%)^{*}$ & $12,336(86.5 \%)$ & $13,322(84.0 \%)$ \\
\hline Rural & $6333(13.6 \%)$ & $1910(11.5 \%)^{*}$ & $1907(13.4 \%)$ & $2516(15.9 \%)$ \\
\hline \multicolumn{5}{|c|}{ Number of unique medications (based on drug name) in last year } \\
\hline Median (IQR) & $11(7-15)$ & $11(7-15)$ & $11(7-15)$ & $10(6-15)$ \\
\hline \multicolumn{5}{|l|}{ Hospitalizations within the last year } \\
\hline Number with one or more hospitalizations & $17,601(37.7 \%)$ & $5745(34.7 \%)^{*}$ & $5097(35.7 \%)^{*}$ & $6759(42.6 \%)$ \\
\hline \multicolumn{5}{|l|}{ Emergency visits within the last year } \\
\hline 0 & $15,442(33.1 \%)$ & $6093(36.8 \%)^{*}$ & $5036(35.3 \%)^{*}$ & $4313(27.2 \%)$ \\
\hline 1 & $12,814(27.4 \%)$ & $4453(26.9 \%)$ & $3862(27.1 \%)$ & $4499(28.4 \%)$ \\
\hline $2-4$ & $14,656(31.4 \%)$ & $4818(29.1 \%)^{*}$ & $4261(29.9 \%)^{*}$ & $5577(35.2 \%)$ \\
\hline $5+$ & $3783(8.1 \%)$ & $1199(7.2 \%)$ & $1107(7.8 \%)$ & $1477(9.3 \%)$ \\
\hline \multicolumn{5}{|l|}{ Physician office visits within the last year } \\
\hline Median (IQR) & $3(2-5)$ & $3(1-5)$ & $3(1-5)$ & $3(2-5)$ \\
\hline \multicolumn{5}{|l|}{ Specialist visits within 3 months } \\
\hline Psychiatrists & $8205(17.6 \%)$ & $2542(15.3 \%)^{*}$ & $2378(16.7 \%)^{*}$ & $3285(20.7 \%)$ \\
\hline Neurologists & $3543(7.6 \%)$ & $1386(8.4 \%)$ & $995(7.0 \%)$ & $1162(7.3 \%)$ \\
\hline Geriatrician & $7340(15.7 \%)$ & $2584(15.6 \%)$ & $2301(16.1 \%)$ & $2455(15.5 \%)$ \\
\hline \multicolumn{5}{|l|}{ Charlson morbidity index } \\
\hline No hospitalization & $20,597(44.1 \%)$ & $7758(46.8 \%)^{*}$ & $6543(45.9 \%)^{*}$ & $6296(39.7 \%)$ \\
\hline 0 & $5773(12.4 \%)$ & $2102(12.7 \%)$ & $1736(12.2 \%)$ & $1935(12.2 \%)$ \\
\hline 1 & $7854(16.8 \%)$ & $2655(16.0 \%)$ & $2351(16.5 \%)$ & $2848(18.0 \%)$ \\
\hline 2 & $4816(10.3 \%)$ & $1668(10.1 \%)$ & $1447(10.1 \%)$ & $1701(10.7 \%)$ \\
\hline $3+$ & $7655(16.4 \%)$ & $2380(14.4 \%)^{*}$ & $2189(15.3 \%)^{*}$ & $3086(19.5 \%)$ \\
\hline \multicolumn{5}{|c|}{ Concomitant psychotropic use during continuous use of antipsychotic } \\
\hline Antidepressants & $31,243(66.9 \%)$ & $11,024(66.6 \%)$ & $9716(68.1 \%)$ & $10,503(66.2 \%)$ \\
\hline Benzodiazepine & $18,711(40.1 \%)$ & $6200(37.4 \%)^{*}$ & $5614(39.4 \%)$ & $6897(43.5 \%)$ \\
\hline Mood stabilizer & $3913(8.4 \%)$ & $1262(7.6 \%)$ & $1152(8.1 \%)$ & $1499(9.4 \%)$ \\
\hline Stimulants & $178(0.4 \%)$ & $47(0.3 \%)$ & $59(0.4 \%)$ & $72(0.5 \%)$ \\
\hline Cognitive enhancers & $22,797(48.8 \%)$ & $8706(52.6 \%)^{*}$ & $7268(50.9 \%)^{*}$ & $6823(43.0 \%)$ \\
\hline \multicolumn{5}{|l|}{ Prescriber of initial antipsychotic prescriptions } \\
\hline Missing & $1192(2.6 \%)$ & $439(2.7 \%)$ & $319(2.2 \%)$ & $434(2.7 \%)$ \\
\hline Other & $8048(17.2 \%)$ & $3025(18.2 \%)^{*}$ & $2525(17.7 \%)^{*}$ & $2498(15.8 \%)$ \\
\hline General practitioner & $37,461(80.2 \%)$ & $13,103(79.1 \%)$ & $11,424(80.1 \%)$ & $12,934(81.5 \%)$ \\
\hline \multicolumn{5}{|l|}{ Equivalent daily doses of antipsychotic dispensed } \\
\hline Mean (SD) & $106.69 \pm 213.38$ & $18.07 \pm 6.08 *$ & $45.79 \pm 9.74 *$ & $253.96 \pm 317.26$ \\
\hline Median (IQR) & $46(20-100)$ & $20(11-23)^{*}$ & $50(40-50)^{*}$ & $150(100-287)$ \\
\hline \multicolumn{5}{|l|}{ Psychosis co-diagnosis } \\
\hline Schizophrenia & $740(1.6 \%)$ & $153(0.9 \%)^{*}$ & $160(1.1 \%)^{*}$ & $427(2.7 \%)$ \\
\hline Bipolar disorder & $395(0.8 \%)$ & $73(0.4 \%)^{*}$ & $99(0.7 \%)$ & $223(1.4 \%)$ \\
\hline
\end{tabular}


Table 1 continued

\begin{tabular}{lllll}
\hline & $\begin{array}{l}\text { Overall } \\
N=46,695\end{array}$ & \multicolumn{2}{l}{ Index equivalent daily dose } \\
\cline { 3 - 5 } & & $\begin{array}{l}\text { Low }(\leq 25 \mathrm{mg}) \\
N=16,563\end{array}$ & $\begin{array}{l}\text { Medium }(>25-74 \mathrm{mg}) \\
N=14,266\end{array}$ & $\begin{array}{l}\text { High }(>74 \mathrm{mg}) \\
N=15,866\end{array}$ \\
\hline Schizophrenia or bipolar disorder & $1064(2.3 \%)$ & $214(1.3 \%)^{*}$ & $248(1.7 \%)^{*}$ & $602(3.8 \%)$ \\
\hline
\end{tabular}

$S D$ standard deviation, $I Q R$ interquartile range

* Denotes significant difference $(p<0.05)$ when compared to other equivalent daily dosing groups

Table 2 Antipsychotic drug groups stratified by initial equivalent daily dose of chlorpromazine

\begin{tabular}{|c|c|c|c|c|}
\hline \multirow[t]{2}{*}{ Antipsychotic drug group } & \multirow{2}{*}{$\begin{array}{l}\text { Total } \\
N=46,695\end{array}$} & \multicolumn{3}{|c|}{ Index equivalent daily dose } \\
\hline & & $\begin{array}{l}\text { Low }(\leq 25 \mathrm{mg}) \\
N=16,563\end{array}$ & $\begin{array}{l}\text { Medium }(>25-74 \mathrm{mg}) \\
N=14,266\end{array}$ & $\begin{array}{l}\text { High }(>74 \mathrm{mg}) \\
N=15,866\end{array}$ \\
\hline Aripiprazole & $184(0.4 \%)$ & $25(0.2 \%)$ & $110(0.8 \%)$ & $49(0.3 \%)$ \\
\hline Chlorpromazine & $114(0.2 \%)$ & $31(0.2 \%)$ & $24(0.2 \%)$ & $59(0.4 \%)$ \\
\hline Haloperidol & $2581(5.5 \%)$ & $104(0.6 \%)$ & $746(5.2 \%)$ & $1731(10.9 \%)$ \\
\hline Loxapine & $107(0.2 \%)$ & $12(0.1 \%)$ & $29(0.2 \%)$ & $66(0.4 \%)$ \\
\hline Methotrimeprazine & $328(0.7 \%)$ & $109(0.7 \%)$ & $104(0.7 \%)$ & $115(0.7 \%)$ \\
\hline Olanzapine & $4937(10.6 \%)$ & $58(0.4 \%)$ & $349(2.4 \%)$ & $4530(28.6 \%)$ \\
\hline Quetiapine & $18,902(40.5 \%)$ & $10,922(65.9 \%)$ & $6156(43.2 \%)$ & $1824(11.5 \%)$ \\
\hline Risperidone & $16,023(34.3 \%)$ & $5246(31.7 \%)$ & $6297(44.1 \%)$ & $4480(28.2 \%)$ \\
\hline Other antipsychotic & $1647(3.5 \%)$ & $54(0.3 \%)$ & $352(2.5 \%)$ & $1241(7.8 \%)$ \\
\hline More than one antipsychotic & $1872(4.0 \%)$ & $\leq 5$ & $96-100$ & $1771(11.2 \%)$ \\
\hline
\end{tabular}

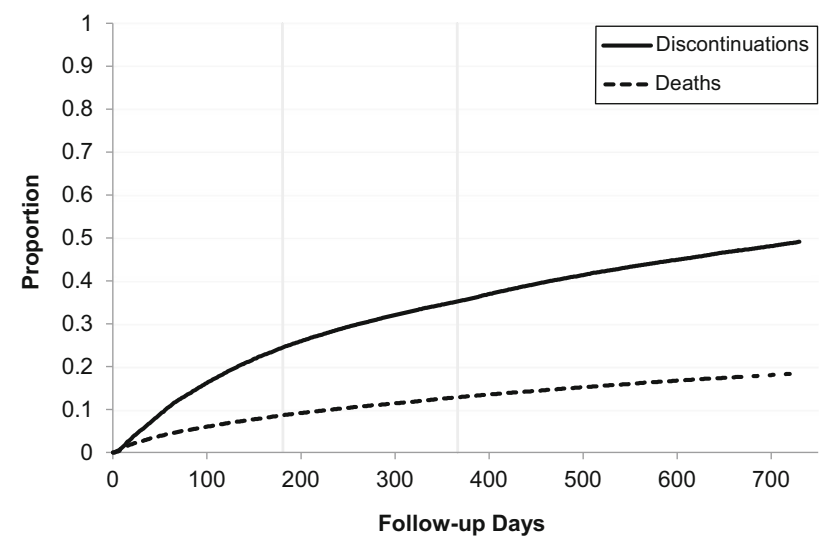

Fig. 1 Proportion of cohort $(N=46,695)$ of elderly dementia patients newly initiated on antipsychotic treatment that discontinued treatment or died during the 2-year study period

\section{Discussion}

In this population-based study, we found that just over half of elderly dementia patients treated with antipsychotics were persistent to therapy over our 2-year study period. Furthermore, we found that persistence to antipsychotic

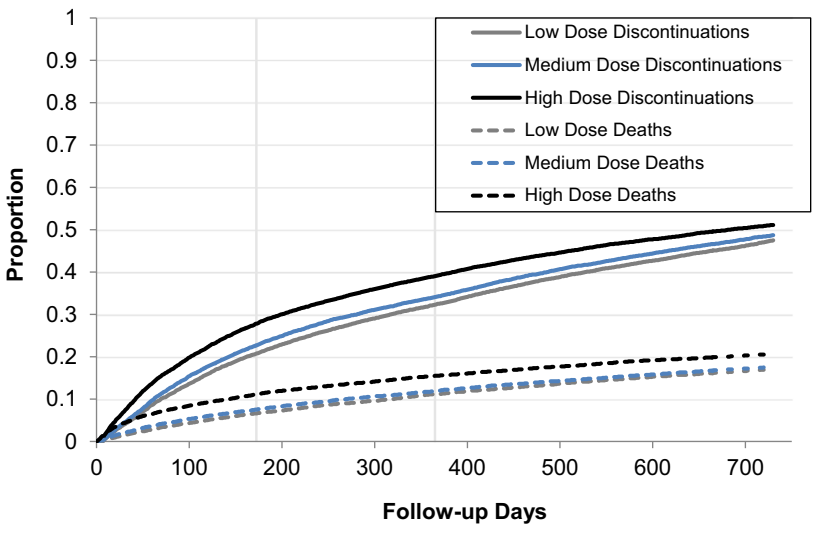

Fig. 2 Proportion of cohort $(N=46,695)$ of elderly dementia patients newly initiated on antipsychotic treatment that discontinued treatment or died during the 2-year study period, stratified by initial antipsychotic dose. Dose divisions of low $(<26 \mathrm{mg} /$ day $)$, medium (26-74 mg/day), and high (>74 mg/day) were determined from tertiles of initial doses and are given in chlorpromazine equivalent daily doses

therapy was lower when patients were initiated on higher doses of medication in both our primary analysis and sensitivity analysis. 
These findings are similar to those identified in other studies examining the persistence and adherence of antipsychotics among schizophrenia and bipolar patients, and are also similar to those observed among some other chronic medications used in the elderly such as statins [10, 14, 23]. In a study by Valenstein et al. [10] examining the adherence to antipsychotics among schizophrenia patients, $40 \%$ of patients were found to be poorly adherent to therapy, while a study by Sajatovic et al. [14] of antipsychotic adherence among bipolar patients found $48.1 \%$ of individuals were either partially adherent or non-adherent. These discontinuations are generally attributed in part to the serious side effects associated with antipsychotic treatment, and whether these trends are found amongst the elderly dementia patient population is a question for future studies [24].

When using antipsychotics to treat schizophrenia and bipolar patients, clinicians aim to encourage medication adherence, while in managing BPSD, clinical practice guidelines and governmental advisories favor short-term use of antipsychotics due to the potential serious harm of these products in this population $[8,9,15]$. Therefore our finding that the majority of patients were persistent to their treatments over the 2-year study period is concerning. With the widespread use of antipsychotics among the elderly dementia patient population, this finding raises questions about why so many patients were kept on antipsychotic therapy well beyond recommended durations, and highlights the need for further research on antipsychotic treatments in this population. Furthermore, it is additionally concerning that just over $40 \%$ of the cohort was concomitantly being treated with benzodiazepines, given the dangers associated with their use among the elderly and frail and their possible association with dementia and cognitive decline [25-27]. The risk of falls and fractures as well as more serious adverse events is likely to be heightened amongst the individuals in our study due to their treatment with both antipsychotics and benzodiazepines, and thus is a troubling finding from our study that should not be overlooked by clinicians.

Our study also demonstrates the potential relationship between persistence and initial dose of antipsychotic administered. We found that over a third of the cohort began treatment on a high dose of antipsychotic, which is concerning as the general approach to BPSD with antipsychotics is to initiate on low doses and slowly increase over time [15]. We also found that there was a small but significant difference between persistence patterns by dose groupings, with more frequent discontinuations among those initiating higher doses. It is possible that those on higher doses experienced greater side effects while on treatment and this prompted discontinuation, although our current study was not able to assess the reason for treatment discontinuation.
However, one must also consider the possibility that clinicians that are prescribing the higher-dose antipsychotics are aware of the safety risks associated with the medication use and thus are discontinuing treatment more rapidly than in patients initiated on lower doses. We believe that this possibility bears further consideration, and presents itself as a future avenue for research. It is also possible that patient differences between the dose groups could explain this finding. Our comparison of the characteristics of antipsychotic users between dose groups (Table 1) shows that these patients differ on a number of characteristics, including age, concomitant cognitive enhancer use, and psychiatrist visits within the past 3 months. While the differences seen were statistically significant, the relative differences between the characteristics of the groups were generally fairly small, suggesting that the patient populations were overall fairly similar.

An important point to consider with regard to medication persistence among the dementia patient population is the variation in the ability to self-manage treatments and the patient's capacities to make decisions. The majority of patients with dementia may have some impairment that can affect their ability to receive treatment, with a worsening of these impairments as the disease progresses [28]. This often results in family members or other caregivers becoming responsible for the medication management of dementia patients, with physicians often advised to take more active monitoring roles [29]. This also further highlights the need to explore why patients were maintained on therapy, as physicians are often much more active in patient medication management $[15,29]$.

Some limitations of this study do bear consideration. We were unable to determine the reason for discontinuation of antipsychotic therapy in our study and can thus only speculate on the role of side effects. As such this presents itself as a future avenue for further research, particularly if awareness amongst clinicians of the side effects prompted the discontinuations as mentioned earlier. One other limitation of this study is that it used only the prescription records for patients, and therefore there is no way to ensure that patients were in fact taking the medication that they were prescribed. However, by restricting our population to those individuals who were dispensed more than one prescription, we have increased the likelihood that there were active users of these medications. Furthermore, we were not able to identify whether discontinuation was driven by the initial dose given or rather by the maximum dose given to patients.

\section{Conclusions}

Our study demonstrates a high level of persistence to antipsychotic therapy among elderly dementia patients over a 2-year period, despite recommendations against the 
long-term use of these drugs for these patients. Furthermore, our findings show that higher doses of these drugs may be associated with more frequent discontinuations, possibly due to the role of side effects. Our findings highlight the need for further research into the usage and duration of antipsychotic therapy among the elderly, particularly due to the potential serious side effects associated with use of these medications.

Acknowledgments We thank Brogan Inc., Ottawa for use of their Drug Product and Therapeutic Class Database. Parts of this material are based on data and information compiled and provided by CIHI. However, the analyses, conclusions, opinions, and statements expressed herein are those of the author, and not necessarily those of CIHI.

Author contributions Gavin Mast was responsible for conception and design of the study, data acquisition, analysis and interpretation, drafting the manuscript, and final approval of the manuscript. Kimberly Fernandes was responsible for data acquisition, analysis and interpretation of data, critical revision of the manuscript and final approval of the manuscript. Mina Tadrous was responsible for conception and design of the study, data acquisition, analysis and interpretation, critical revision of the manuscript, and final approval of the manuscript. Dianna Martins was responsible for conception and design of the study, data acquisition, analysis and interpretation, critical revision of the manuscript, and final approval of the manuscript. Nathan Herrmann was responsible for conception and design of the study, data interpretation, critical revision of the manuscript, and final approval of the manuscript. Tara Gomes was responsible for conception and design of the study, data acquisition, analysis and interpretation, critical revision of the manuscript, and final approval of the manuscript.

\section{Compliance with Ethical Standards}

Funding This study was funded by the Ontario Drug Policy Research Network which is funded by grants from the Ontario Ministry of Health and Long-Term Care (MOHLTC), and supported by the Institute for Clinical Evaluative Sciences (ICES). The opinions, results, and conclusions reported in this paper are those of the authors and are independent from the funding sources. No endorsement by ICES or the Ontario MOHLTC is intended or should be inferred.

Conflict of interest Tara Gomes has received grants from the Ontario Ministry of Health and Long Term Care. Nathan Herrmann has received grants from Lundbeck, and consulting fees from Astellas and AbbVie. Gavin Mast, Kimberley Fernandes, Mina Tadrous, and Diana Martins have no conflicts of interest to declare that might be relevant to this manuscript.

Open Access This article is distributed under the terms of the Creative Commons Attribution-NonCommercial 4.0 International License (http://creativecommons.org/licenses/by-nc/4.0/), which permits any noncommercial use, distribution, and reproduction in any medium, provided you give appropriate credit to the original author(s) and the source, provide a link to the Creative Commons license, and indicate if changes were made.

\section{Appendix}

See Figs. 3 and 4.

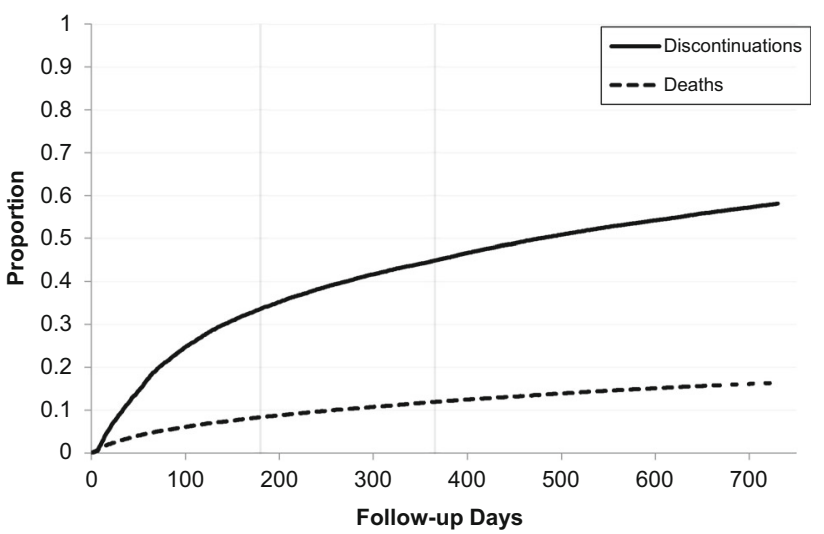

Fig. 3 Proportion of cohort $(N=46,695)$ of elderly dementia patients newly initiated on antipsychotic treatment that discontinued treatment or died during the 2-year study period using alternate 1.5 times day supplied definition for continuous use

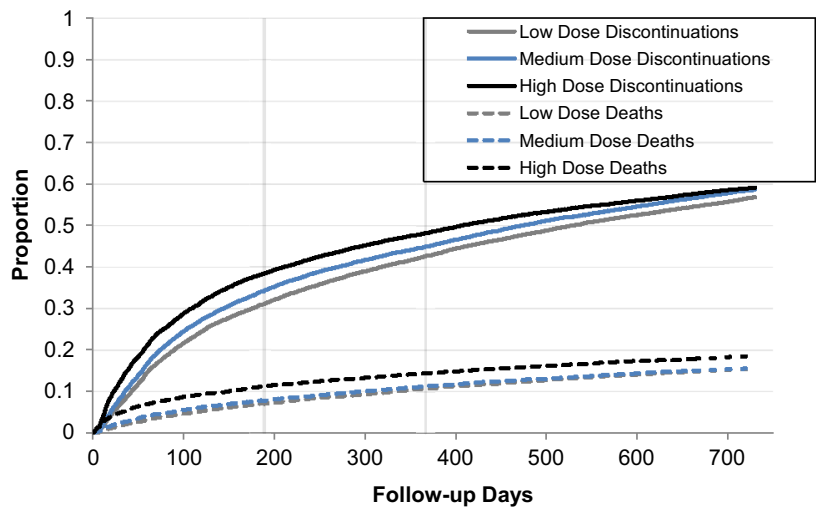

Fig. 4 Proportion of cohort $(N=46,695)$ of elderly dementia patients newly initiated on antipsychotic treatment that discontinued treatment or died during the 2-year study period using alternate 1.5 times day supplied definition for continuous use, stratified by initial antipsychotic dose. Dose divisions of low ( $<26 \mathrm{mg} /$ day), medium (26-74 mg/day), and high ( $>74 \mathrm{mg} /$ day) were determined from tertiles of initial doses and are given in chlorpromazine equivalent daily doses

\section{References}

1. Gill SS, Bronskill SE, Normand SLT, Anderson GM, Sykora K, Lam K, Bell CM, Lee PE, Fischer HD, Herrmann N, Gurwitz JH, Rochon PA. Antipsychotic drug use and mortality in older adults with dementia. Ann Intern Med. 2007;146:775-86.

2. Schneider LS, Dagerman K, Insel PS. Efficacy and adverse effects of atypical antipsychotics for dementia: meta-analysis of randomized, placebo-controlled trials. Am J Geriat Psychiat. 2006;14:191-210.

3. Ontario Drug Policy Research Network, Antipsychotic use in the elderly 2015. http://odprn.ca/antipsychotics-in-the-elderly/. Accessed 8 June 2015.

4. Ray WA, Chung CP, Murray KT, Hall K, Stein CM. Atypical antipsychotic drugs and the risk of sudden cardiac death. N Engl J Med. 2009;360:225-35. 
5. Hennessy S, Bilker WB, Knauss JS, Margolis DJ, Kimmel SE, Reynolds RF, Glasser DB, Morrison MF, Strom BL. Cardiac arrest and ventricular arrhythmia in patients taking antipsychotic drugs: cohort study using administrative data. Brit Med J. 2002;325:1070.

6. Rummel-Kluge C, Komossa K, Schwarz S, Hunger H, Schmid F, Kissling W, Davis JM, Leucht S. Second-generation antipsychotic drugs and extrapyramidal side effects: a systematic review and meta-analysis of head-to-head comparisons. Schizophrenia Bull. 2012;38:167-77.

7. Jalbert JJ, Eaton CB, Miller SC, Lapane KL. Antipsychotic use and the risk of hip fracture among older adults afflicted with dementia. J Am Med Dir Assoc. 2010;11:120-7.

8. Atypical antipsychotic drugs and dementia: advisories, warnings and recalls for health professionals. Canadian Adverse Drug Reaction Monitoring Program, Health Canada. Ottawa; 2005. Available from: http://www.healthycanadians.gc.ca/recall-alertrappel-avis/hc-sc/2005/14307a-eng.php.

9. Antipsychotics, conventional and atypical. United States Food and Drug Association. Silver Spring; 2008. Available from: http://www.fda.gov/Safety/MedWatch/SafetyInformation/Safety AlertsforHumanMedicalProducts/ucm110212.htm.

10. Valenstein M, Blow FC, Copeland LA, McCarthy JF, Zeber JE, Gillon L, Bingham CR, Stavenger T. Poor antipsychotic adherence among patients with schizophrenia: medication and patient factors. Schizophr Bull. 2004;30:255-64.

11. Ascher-Svanum H, Zhu B, Faries DE, Lacro JP, Dolder CR, Peng $\mathrm{X}$. Adherence and persistence to typical and atypical antipsychotics in the naturalistic treatment of patients with schizophrenia. Patient Prefer Adherence. 2008;2:67-77.

12. Menzin J, Boulanger L, Friedman M, Mackell J, Lloyd JR. Treatment adherence associated with conventional and atypical antipsychotics in a large state Medicaid program. Psychiatr Serv. 2014;54:719-23.

13. Dolder CR, Lacro JP, Dunn LB, Jeste DV. Antipsychotic medication adherence: is there a difference between typical and atypical agents? Am J Psychiatry. 2002;159:103-8.

14. Sajatovic M, Valenstein M, Blow FC, Ganoczy D, Ignacio RV. Treatment adherence with antipsychotic medications in bipolar disorder. Bipolar Disord. 2006;8:232-41.

15. Best practice guideline for accommodating and managing behavioral and psychological symptoms of dementia in residential care. Ministry of Health. British Columbia; 2012. Available from: http://www.health.gov.bc.ca/library/publications/year/2012/ bpsd-guideline.pdf.

16. Rochon PA, Stukel TA, Bronskill SE, Gomes T, Sykora K, Wodchis WP, Hillmer M, Kopp A, Gurwitz JH, Anderson GM. Variation in nursing home antipsychotic prescribing rates. Arch Intern Med. 2007;167:676-83.
17. Schneeweiss S, Patrick AR, Stürmer T, Brookhart MA, Avorn J, Maclure M, Rothman KJ, Glynn RJ. Increasing levels of restriction in pharmacoepidemiologic database studies of elderly and comparison with randomized trial results. Med Care. 2007;45:S131-42.

18. Gomes T, Juurlink DN, Mamdani MM. Comparative adherence to oxybutynin or tolterodine among older patients. Eur J Clin Pharmacol. 2012;68:97-9.

19. Vigod SN, Kurdyak PA, Dennis CL, Gruneir A, Newman A, Seeman MV, Rochon PA, Anderson GM, Grigoriadis S, Ray JG. Maternal and newborn outcomes among women with schizophrenia: a retrospective population-based cohort study. Brit J Obstet Gynaecol. 2014;121:566-74.

20. Woods SW. Chlorpromazine equivalent doses for the newer atypical antipsychotics. J Clin Psychiatry. 2003;64:663-7.

21. Mamdani M, Sykora K, Li P, Normand SL, Streiner DL, Austin PC, Rochon PA, Anderson GM. Reader's guide to critical appraisal of cohort studies: 2. Assessing potential for confounding. Br Med J. 2005;330:960-2.

22. Gray RJ. A class of K-sample tests for comparing the cumulative incidence of a competing risk. Ann Stat. 1988;16:1141-54.

23. Carter AA, Gomes T, Camacho X, Juurlink DN, Shah BR, Mamdani MM. Risk of incident diabetes among patients treated with statins: population based study. Br Med J. 2013;346:f2610.

24. Ritsner M, Ponizovsky A, Endicott J, Nechamkin Y, Rauchverger B, Silver H, Modai I. The impact of side-effects of antipsychotic agents on life satisfaction of schizophrenia patients: a naturalistic study. Eur Neuropsychopharmacol. 2002;12:31-8.

25. Landi F, Onder G, Cesari M, Barillaro C, Russo A, Bernabei R. Psychotropic medications and risk for falls among communitydwelling frail older people: an observational study. J Gerontol A Biol Sci Med Sci. 2005;60:622-6.

26. de Gage SB, Bégaud B, Bazin F, Verdoux H, Dartigues JF, Pérès K, Kurth T, Pariente A. Benzodiazepine use and risk of dementia: prospective population based study. Br Med J. 2012;345:e6231.

27. Mura T, Proust-Lima C, Akbaraly T, Amieva H, Tzourio C, Chevassus H, Picot MC, Jacqumin-Gadda H, Berr C. Chronic use of benzodiazepines and latent cognitive decline in the elderly: results from the Three-city study. Eur Neuropsychopharmacol. 2013;23:212-23.

28. Appelbaum RS. Assessment of patients' competence to consent to treatment. N Engl J Med. 2007;357:1834-40.

29. Hogan DB, Bailey P, Black S, Carswell A, Chertkow H, Clarke B, Cohen C, Fisk JD, Forbes D, Man-Son-Hing M, Lanctôt K, Morgan D, Thorpe L. Diagnosis and treatment of dementia: 4. approach to management of mild to moderate dementia. Can Med Assoc J. 2008;179:787-93. 